\title{
Existence of Quasi-Convex Solution in Nonlinear Programming
}

\author{
Okpara, Patrick* Effor Theresa \\ Department of Industrial Mathematics and Applied Statistics, Ebonyi State University, Abakaliki
}

\begin{abstract}
Herein is characterized the solution of quasiconvex optimization in nonlinear programming problem using Kuhn-tucker theorem for problems with inequality constraints. The purpose of this paper is to present sufficient conditions using Kuhn-Tucker first order conditions to identify optima solution of inequality constrained optimization problems.
\end{abstract}

Keywords: Qausiconvex, Nonlinear Programming, Optimization, Optimal Solution, Kuhn-Tucker, Convexity.

\section{Introduction}

The nonlinear quasiconvex optimization problem may be stated as follows:

$$
\text { minimize } f(x)
$$

Such that $\underline{x} \in\left\{\underline{x} \in \mathbb{R}^{n}: g(\underline{x}) \leq 0, A \underline{x}=\underline{b}\right\} \subset \mathbb{R}^{n}$ where $g: \mathbb{R}^{n} \rightarrow \mathbb{R}^{m}, h: \mathbb{R}^{n} \rightarrow \mathbb{R}^{k}$

We cannot discuss quasiconvex optimization without the concept of convexity. In this case, convexity property is very essential in optimization. Most algorithms that are used in computing the minimum point or maximum point if they exist are point to point maps and the solution they generate are extremum points. Minimization problems in convex function plays an important role in identifying global minima.

Let $f: S \rightarrow \mathbb{R} \subset \mathbb{R}^{n}$, then $f$ is said to be quasiconvex on $S$ if

$$
f\left(\alpha x_{1}+(1-\alpha) x_{2}\right) \leq \alpha f\left(x_{1}\right)+(1-\alpha) f\left(x_{2}\right) \forall x_{1}, x_{2} \in S, \alpha \in(0,1)
$$

In considering a quasiconvex function, we mean that the function contains a convex constraint sets and a quasiconvex objective function.

Let $f: S \rightarrow \mathbb{R} \subset \mathbb{R}^{n}$, then $f$ is said to be convex on $S$ if

$$
f\left[\alpha x_{1}+(1-\alpha) x_{2}\right] \leq \operatorname{maximum}\left\{f\left(x_{1}\right), f\left(x_{2}\right)\right\} \text { for each } \alpha \in(0,1)
$$

In quasiconvex optimization, $f$ is said to be quasiconvex if whenever $f\left(x_{2}\right) \geq f\left(x_{1}\right)$, then $f\left(x_{2}\right)$ is greater than or equal to $f$ at all convex combinations of $x_{1}$ and $x_{2}$.

\section{Theorem 2.1}

The function $f$ is said to be quasiconvex if and only if for all $x_{1}, x_{2} \in S$ and $\alpha \in(0,1)$

Proof

$$
f\left(\alpha x_{1}+(1-\alpha) x_{2}\right) \leq \max \left\{f\left(x_{1}\right), f\left(x_{2}\right)\right\}
$$

Suppose that $f$ is quasiconvex and let $x_{1} \cdot x_{2} \in S$ and $\alpha \in(0,1)$ and maximum $\left\{f\left(x_{1}\right), f\left(x_{2}\right)\right\} \leq \beta$. By convexity of $S$ we have $x=\alpha x_{1}+(1-\alpha) x_{2}, x \in S$. Furthermore, by quasiconvexity of $f$ we have $f(x) \leq$ $\max \left\{f\left(x_{1}\right), f\left(x_{2}\right)\right\} \leq \beta$. Hence $x \in S$ and thus $S$ is convex.

Conversely, suppose that $f\left(\alpha x_{1}+(1-\alpha) x_{2}\right) \leq \max \left\{f\left(x_{1}\right), f\left(x_{2}\right)\right\}$ for all $x_{1}, x_{2} \in S$ and $\alpha \in(0,1)$ where $x=\alpha x_{1}+(1-\alpha) x_{2}$ then $\beta=\max \left\{f\left(x_{1}\right), f\left(x_{2}\right)\right\}$ since $\beta$ is arbitrarily chosen the $f$ is quasiconvex.

\section{Theorem 2.2}

Let $f: S \rightarrow \mathbb{R}^{n}$, if $f$ is convex on $S$ then it is also quasiconvex on $S$

Proof

Quasiconvexity is the generalization of convexity in that it shows that the set of all quasiconvex functions contain the set of all convex functions.

Suppose that $f$ is convex, then for all $x_{1}, x_{2} \in S$ and $\alpha \in(0,1)$ we have

$$
f\left(\alpha x_{1}+(1-\alpha) x_{2}\right) \leq \alpha f\left(x_{1}\right)+(1-\alpha) f\left(x_{2}\right)
$$

Theorem 2.3

$$
\leq \alpha \max \left\{f\left(x_{1}\right), f\left(x_{2}\right)\right\}+(1-\alpha) \max \left\{f\left(x_{1}\right), f\left(x_{2}\right)\right\}=\max \left\{f\left(x_{1}\right), f\left(x_{2}\right)\right\}
$$

Let $f: S \rightarrow \mathbb{R}$ be a function where $S \subset \mathbb{R}^{n}$ is convex and open. Then $f$ is a quasi concave function on $S$ if and only if for all $x_{1}, x_{2} \in S$,

$$
f\left(x_{2}\right) \geq f\left(x_{1}\right) \Rightarrow \nabla f\left(x_{1}\right)\left(x_{2}-x_{1}\right) \geq 0
$$

\section{Proof}

Here, we characterize the properties of quasiconvex function using first and second derivatives. Suppose that $f$ is quasiconcave on $S$ and let $x_{1}, x_{2} \in S$ such that $f\left(x_{2}\right) \geq f\left(x_{1}\right)$.

Let $\alpha \in(0,1)$, since $f$ is quasi concave, where

$$
f\left(x_{1}+\alpha\left(x_{2}-x_{1}\right)\right)=f\left((1-\alpha) x_{1}+\alpha x_{2}\right) \geq \min \left\{f\left(x_{1}\right), f\left(x_{2}\right)\right\}=f\left(x_{1}\right)
$$


Therefore, for all $\alpha \in(0,1)$ we have $f\left(x_{1}+\alpha\left(x_{2}-x_{1}\right)-f\left(x_{1}\right)\right)$

Then it converges to

$$
\frac{f\left(x_{1}+\alpha\left(x_{2}-x_{1}\right)\right)-f\left(x_{1}\right)}{\alpha} \geq 0 \text { as } \alpha \rightarrow 0
$$

Therefore

$$
\nabla f\left(x_{1}\right) f\left(x_{2}-x_{1}\right)
$$

$$
\nabla f\left(x_{1}\right)\left(x_{2}-x_{1}\right) \geq 0
$$

Conversely, assume that for all $x_{1}, x_{2} \in S$ such that $f\left(x_{2}\right) \leq f\left(x_{1}\right)$ we have

without loss of generality, we have

$$
\nabla f\left(x_{1}\right)\left(x_{2}-x_{1}\right) \geq 0
$$

Then for any $\alpha \in(0,1)$ we have

$$
f\left(x_{1}\right)=\min \left\{f\left(x_{1}\right), f\left(x_{2}\right)\right\}
$$

$$
f\left\{(1-\alpha) x_{1}+\alpha x_{2}\right\} \geq \min \left\{f\left(x_{1}\right), f\left(x_{2}\right)\right\}
$$

Which proves the quasi concavity of $f$. The second derivative characterization of quasiconvexity is implied.

\section{Result of Quasi Concavity}

The result of quasi concavity is the Kuhn-Tucker condition. It shows that the Kuhn-Tucker first order conditions are sufficient to identify the global optima of the inequality constrained maximization problem if all the functions involved are quasi concave.

\section{Theorem 3.1}

Let $f$ and $h_{i}(i=1, \ldots, k)$ be $C^{\prime}$ quasi concave functions mapping $P \subset \mathbb{R}^{n}$ into $\mathbb{R}$ where $P$ is open and convex.

$$
K:=\left\{x \in P \mid h_{i}(x) \geq 0, i=1, \ldots, k\right\}
$$

Suppose there exist $x^{*} \in K$ and $\alpha \in \mathbb{R}^{K}$ such that the Kuhn-Tucker first order conditions are met:

$$
\begin{aligned}
& {[K T-1] \nabla f\left(x^{*}\right)+\sum_{i=1}^{k} \alpha_{i} \nabla h_{i}\left(x^{*}\right)=0} \\
& {[K T-2] \alpha_{i} \geq 0, \alpha_{i} h_{i}\left(x^{*}\right)=0,1, \ldots, k}
\end{aligned}
$$

Then $x^{*}$ maximizes $f$ over $K$ provided at least one of the following conditions hold

$$
\begin{gathered}
{\left[Q_{c}-1\right] \nabla f\left(x^{*}\right) \neq 0} \\
{\left[Q_{c}-2\right] f \text { is concave }}
\end{gathered}
$$

Suppose $\nabla f\left(x^{*}\right) \neq 0$ then there exists $w \in \mathbb{R}^{n}$ such that $\nabla f\left(x^{*}\right) w<0$ let $z=x^{*}+w$, we have $\nabla f\left(x^{*}\right)(z-$ $\left.x^{*}\right)<0$ choose any $x_{1} \in K$, for $\alpha \in(0,1)$

Let $x_{1}(\alpha)=(1-\alpha) x_{1}+\alpha z$ and $x(\alpha)=(1-\alpha) x^{*}+\alpha z$. Fix any $\alpha \in(0,1)$ we have

$$
\nabla f\left(x^{*}\right)\left(x(\alpha)-x^{*}\right)-\alpha \nabla f\left(x^{*}\right)\left(z-x^{*}\right)<0
$$

From the definition of $z$ we have

$$
\nabla f\left(x^{*}\right)\left(x_{1}(\alpha)-x(\alpha)\right)=(1-\alpha) \nabla f\left(x^{*}\right)\left(x_{1}-x^{*}\right) \leq 0
$$

Adding (3.1) and (3.2) we have

$$
\nabla f\left(x^{*}\right)\left(x_{1}(\alpha)-x^{*}\right)<0
$$

By theorem of Kuhn-Tucker $f\left(x_{1}(t)\right)<f\left(x^{*}\right)$ since this holds for any $\alpha \in(0,1)$ taking limit as $\alpha \rightarrow 1$, we have $f(x)<f\left(x^{*}\right)$ and since $x_{1} \in k$ was chosen arbitrarily, it implies that $x^{*}$ is a global maximum of $f$ on $K$.

\section{Application of Kuhn-Tucker Methods}

The theorem of Kuhn-Tucker provides a characterization of the behavior of the objective function and the constraint functions at the local optima of inequality constrained optimization problems. The conditions it describes may be the first order necessary conditions for local optima in the problems.

We consider the practical application as follows:

$$
\begin{array}{ll}
\text { Minimize } & 6\left(x_{1}-10\right)^{2}+4\left(x_{2}^{2}-12.5\right)^{2} \\
\text { Subject to } & x_{1}^{2}+\left(x_{2}-5\right)^{2} \leq 50 \\
& x_{1}^{2}+3 x_{2} \leq 200 \\
& \left(x_{1}-6\right)^{2}+x_{2}^{2} \leq 37
\end{array}
$$

Now we have the following

$$
\begin{aligned}
& f(x)=6\left(x_{1}-10\right)^{2}+4\left(x_{2}-12.5\right)^{2} \\
& h_{1}(x)=x_{1}^{2}+\left(x_{2}-5\right)^{2}-50 \\
& h_{2}(x)=x_{1}^{2}+3 x_{2}^{2}-200 \\
& h_{3}(x)=\left(x_{1}-6\right)^{2}+x_{2}^{2}-37 \\
& \nabla f(x)=\left[\begin{array}{l}
12\left(x_{1}-10\right) \\
8\left(x_{2}-12.5\right)
\end{array}\right]
\end{aligned}
$$




$$
\nabla h_{1}(x)=\left[\begin{array}{l}
2 x_{1} \\
2\left(x_{2}-5\right)
\end{array}\right], \quad \nabla h_{2}(x)=\left[\begin{array}{l}
2 x \\
6 x_{2}
\end{array}\right], \quad \nabla h_{3}(x)=\left[\begin{array}{l}
2\left(x_{1}-6\right) \\
2 x_{2}
\end{array}\right]
$$

We want to solve

$$
\nabla f(x)+\sum_{i=1}^{3} \lambda_{i} \nabla h_{i}(x)=0
$$

We obtain the solution as follows:

$$
\lambda^{*}=\left(\lambda_{1}^{*}, \lambda_{2}^{*}, \lambda_{3}^{*}\right)=(2,0,4)
$$

This is the optimal solution of the above problem.

\section{Conclusion}

In this paper, an attempt has been made to characterize global optimization solution of more general objective functional using convexity assumptions. We were able to obtain that first order conditions are not sufficient to identify global maximum or minimum of quasi concave or convex optimization problems. It was obtained that the Kuhn-Tucker first order conditions are sufficient to identify optima of inequality constrained optimization problems under quasiconvexity restrictions.

\section{References}

[1]. Borwein, J.M. and Lewis, A.S. (2000): Convex Analysis and Nonlinear Optimization (Theory and Examples) Springer-Verlag, New York.

[2]. Boyd, S. and Vandersherghe, L. (2004): Convex Optimization, Cambridge University Press, United Kingdom.

[3]. Horst, R. et al (1990): Global Optimization (deterministic approaches), Springer-Verlag, New York.

[4]. Luenberger, D.G. (1969): Optimization by vector space methods. Wiley, New York.

[5]. Osinuga, I.A. and Bamigbola, O.M. (2004): On Global Optimization for Convex Problems on Abstract Spaces, ABACUS(J. Math. Ass. Nig) 31(2A), $94-98$.

[6]. Osinuga, I.A. (2007): Further Results on the Classes of Convex Functions, J. Nig. Ass. Math. Phys. 11, 515 - 518.

[7]. Zalinescu, C. (2002): Convex Analysis in General Vector Spaces, World Scientific, Singapore. 